\title{
Differential and Converging Molecular Mechanisms of Antidepressants' Action in the Hippocampal Dentate Gyrus
}

\begin{abstract}
Patrícia Patrício 1,2 , António Mateus-Pinheiro' ${ }^{1,2}$, Martin Irmler ${ }^{3}$, Nuno D Alves ${ }^{1,2}$, Ana R Machado-Santos ${ }^{1,2}$, Mónica Morais ${ }^{1,2}$, Joana S Correia ${ }^{1,2}$, Michal Korostynski ${ }^{4}$, Marcin Piechota ${ }^{4}$, Rainer Stoffel ${ }^{5}$, Johannes Beckers ${ }^{3,6}$, João M Bessa ${ }^{1,2}$, Osborne FX Almeida ${ }^{5}$, Nuno Sousa*, ${ }^{*, 2}$ and Luísa Pinto*, 1,2

'Life and Health Sciences Research Institute (ICVS), School of Health Sciences, University of Minho, Braga, Portugal; ${ }^{2}$ CVVS/3B's —PT Government Associate Laboratory, Braga/Guimarães, Portugal; ${ }^{3}$ Institute of Experimental Genetics, Helmholtz Zentrum München, German Research Center for Environmental Health, Neuherberg, Germany; ${ }^{4}$ Department of Molecular Neuropharmacology, Institute of Pharmacology, Polish Academy of Sciences, Kraków, Poland; ${ }^{5}$ Max Planck Institute of Psychiatry, Munich, Germany; ${ }^{6}$ Technical University Munich, Chair of Experimental Genetics, Freising-Weihenstephan, Germany
\end{abstract}

Major depression is a highly prevalent, multidimensional disorder. Although several classes of antidepressants (ADs) are currently available, treatment efficacy is limited, and relapse rates are high; thus, there is a need to find better therapeutic strategies. Neuroplastic changes in brain regions such as the hippocampal dentate gyrus (DG) accompany depression and its amelioration with ADs. In this study, the unpredictable chronic mild stress (UCMS) rat model of depression was used to determine the molecular mediators of chronic stress and the targets of four ADs with different pharmacological profiles (fluoxetine, imipramine, tianeptine, and agomelatine) in the hippocampal DG. All ADs, except agomelatine, reversed the depression-like behavior and neuroplastic changes produced by uCMS. Chronic stress induced significant molecular changes that were generally reversed by fluoxetine, imipramine, and tianeptine. Fluoxetine primarily acted on neurons to reduce the expression of pro-inflammatory response genes and increased a set of genes involved in cell metabolism. Similarities were found between the molecular actions and targets of imipramine and tianeptine that activated pathways related to cellular protection. Agomelatine presented a unique profile, with pronounced effects on genes related to Rho-GTPase-related pathways in oligodendrocytes and neurons. These differential molecular signatures of ADs studied contribute to our understanding of the processes implicated in the onset and treatment of depression-like symptoms.

Neuropsychopharmacology (2015) 40, 338-349; doi:I0.1038/npp.20I4.I76; published online I3 August 20I4

\section{INTRODUCTION}

Major depression is one of the most prevalent neuropsychiatric disorder and is characterized by a multifaceted profile of behavioral deficits, such as depressed mood, anxiety, and cognitive impairments (Bessa et al, 2009b; Villanueva, 2013). Strikingly, a high percentage of patients treated with the currently available therapies do not show full remission (Lang and Borgwardt, 2013) and present treatment resistance (Blier and Blondeau, 2011). Although the pathophysiology of depression is still incompletely understood, dysregulation of monoaminergic systems, neuroplasticity, and immunological responses (Villanueva, 2013; Willner et al, 2013) are considered to contribute to the disease. In addition, alterations in dendritic plasticity and cytogenesis in the hippocampal

*Correspondence: Professor N Sousa or Dr L Pinto, Life and Health Sciences Research Institute (ICVS), School of Health Sciences, University of Minho, Campus de Gualtar, Braga 47I 0-057, Portugal, Tel: +35 I 253 604 947, Fax: +35I 253604 809, E-mail: njcsousa@ecsaude.uminho.pt or luisapinto@ecsaude.uminho.pt

Received II April 20 I4; revised I9 June 20 4; accepted I I July 20 I4; accepted article preview online 18 July 2014 dentate gyrus (DG) are observed in the brains of animal models of depression and depressed patients (Lucassen et al, 2014; Pittenger and Duman, 2008). Importantly, these changes have been implicated in the onset of depressivelike symptoms and in the actions of antidepressants (ADs) in animal models of depression (Bessa et al, 2009a; Mateus-Pinheiro et al, 2013a, b; Surget et al, 2011).

$\mathrm{ADs}$ are generally classified according to their primary pharmacological targets. The first developed ADs were monoamine oxidase inhibitors (MAOi) and tricyclic (TCA) and tetracyclic agents (Li et al, 2012). Second-generation ADs include the selective serotonin reuptake inhibitors (SSRIs), still the most prescribed ADs worldwide, and norepinephrine and serotonin reuptake inhibitors (NSRIs). Atypical ADs include tianeptine whose mechanisms of action are poorly understood but which exert potent neuroplastic effects (McEwen et al, 2010), and agomelatine, an agonist of melatonin receptors (MT1 and MT2) and an antagonist of the $5 \mathrm{HT}_{2 \mathrm{c}}$ receptor that was recently introduced as an $\mathrm{AD}$ (Tardito et al, 2012). Despite their diverse pharmacological profiles, all of these drugs result in similar behavioral outcomes, suggesting overlapping mechanisms of action. Insights into their potentially common molecular 
targets and divergent mechanisms may help develop new treatment strategies that exploit specific properties of each individual drug.

Application of genome-wide analyses of specific brain regions is a potentially valuable approach to study drugspecific molecular targets and to generate information that may facilitate the development of more efficacious treatments. Such approaches have been previously used to investigate the molecular alterations induced by $\mathrm{ADs}$ in the hippocampus and other brain regions of naive animals (Conti et al, 2007; Gaska et al, 2012; Korostynski et al, 2013; Landgrebe et al, 2002; Lee et al, 2010; Sillaber et al, 2008; Surget et al, 2009; Takahashi et al, 2006); interpretation of these studies is, however, limited by the fact that treatment of individuals who do not display signs of depressive-like behavior is not comparable to a pathological context (Cryan and Slattery, 2007). Previous comparable studies relied on measures of a single behavioral index measurement (Drigues et al, 2003; Nakatani et al, 2004), while others using multidimensional animal models of depression focused on a single class of AD (Andrus et al, 2012; Datson et al, 2012; Lisowski et al, 2013; Surget et al, 2009) or two monoaminergic ADs (Malki et al, 2012).

Given the complex nature of depression and the heterogeneous pharmacological profiles of available ADs, we considered it important to undertake a comprehensive comparison of the molecular effects of commonly used ADs, focusing on the effects elicited in the DG of rats exposed to a well-characterized multidimensional animal model of depression, the unpredictable chronic mild stress (uCMS) (Bessa et al, 2009b; Hill et al, 2012). Exposure to uCMS results in animals with behavioral deficits and biometric and neuroplastic changes that match many of those found in patients with major depression (Bessa et al, 2013, 2009b; Hill et al, 2012; Lucassen et al, 2014). Following induction of disease-like symptoms, animals were treated with either fluoxetine, imipramine, tianeptine, or agomelatine, and transcriptome analysis was subsequently performed on their DGs.

\section{MATERIALS AND METHODS}

\section{Animals and Treatments}

Male Wistar rats (200-250 g, 2-month old; Charles River Laboratories) were maintained under standard laboratory conditions (lights on: $0800-2000$ hours; $22{ }^{\circ} \mathrm{C}$, relative humidity of $55 \%$, ad libitum access to food and water). Rats ( $n=8-12 /$ group for behavioral analysis, of which 3 were considered for microarray analysis and 5/6 for morphological analysis) were randomly assigned to one of the eight groups: non-stressed control (CT) + vehicle; stressexposed (uCMS) + vehicle; and uCMS + fluoxetine (FLX)/ imipramine (IMIP)/tianeptine (TIAN)/agomelatine (AGOM). A validated uCMS protocol was applied for 6 weeks, as previously described (Bessa et al, 2009b). During the last 2 weeks of the uCMS, animals were injected intraperitoneally daily with one of the four ADs: fluoxetine $(10 \mathrm{mg} / \mathrm{kg}$ in ultra-pure water; Kemprotec, Middlesborough, UK); imipramine $(10 \mathrm{mg} / \mathrm{kg}$ in $0.9 \%$ saline solution; SigmaAldrich, St Louis, MO, USA); tianeptine (10 mg/kg in $0.9 \%$ saline solution; Kemprotec); and agomelatine $\left(40 \mathrm{mg} / \mathrm{kg}^{1}\right.$ in
$0.5 \%$ hydroxyethylcellulose; Kemprotec). The doses were chosen based on previous studies (Banasr et al, 2006; Bessa et al, 2009a; McEwen et al, 2010). Body weight was monitored weekly (Supplementary Figure S1a). All procedures were carried out in accordance with EU Directive 2010/63/EU and NIH guidelines on animal care and experimentation.

\section{Behavioral Analysis}

Sucrose consumption test (SCT). Anhedonia was assessed for all animals by the SCT 4 and 6 weeks into the uCMS protocol (Figure 1a). Baseline sucrose preference (SP) was determined immediately before the start of the uCMS protocol (three independent trials). Before each trial, rats were food and water deprived for $12 \mathrm{~h}$. For testing, animals were presented with two preweighed bottles, containing water or $2 \%(\mathrm{~m} / \mathrm{v})$ sucrose solution for $1 \mathrm{~h}$. SP was calculated as previously described (Bessa et al, 2009b).

Sweet Drive Test (SDT). The SDT test was used as an additional measure of anhedonic behavior for all animals, as previously described (Mateus-Pinheiro et al, 2014). Briefly, each animal was allowed to explore the SDT box for $10 \mathrm{~min}$ where sweet (Cheerios, Nestlé) or regular pellets (Mucedola 4RF21-GLP) were available. After each trial, preference for sweet pellets was calculated as follows: Preference for sweet pellets $(\%)=$ sweet pellets consumed $(\mathrm{g}) /$ total pellets consumed $(\mathrm{g}) \times 100$. Three SDT trials were conducted during the last week of the uCMS protocol (1 trial every $48 \mathrm{~h}$; Figure 1a). The number of entries into each chamber was used as a measure of exploratory behavior (Supplementary Figure S1b).

Novelty suppressed feeding (NSF) test. Anxiety-like behavior was assessed using the NSF test at the end of the uCMS protocol. Food-deprived $(18 \mathrm{~h})$ animals were placed in an open-field arena for a maximum of $10 \mathrm{~min}$, where a single food pellet was positioned in the center, as previously described (Bessa et al, 2009b). After reaching the pellet, animals were individually returned to their home cage and were allowed to feed for $10 \mathrm{~min}$. The latency to feed in the open-field arena was used as an index of anxiety-like behavior, whereas the food consumption in the home cage provided a measure of appetite drive (Supplementary Figure S1c).

Forced swim test (FST). The FST was performed at the end of the uCMS protocol. Assays were conducted $24 \mathrm{~h}$ after a 5 -min pretest session by placing the rats in glass cylinders filled with water $\left(23^{\circ} \mathrm{C} ; 50 \mathrm{~cm}\right.$ deep $)$ for $5 \mathrm{~min}$. Trials were video-recorded and an increase in immobility time was considered to be a higher degree of depressive-like behavior.

\section{Corticosterone Levels Measurement}

For all animals, corticosterone levels were measured in blood serum using a $\left[{ }^{125} \mathrm{I}\right]$ radioimmunoassay kit (MP Biomedicals, Costa Mesa, CA), according to the manufacturer's instructions. Blood sampling (tail venipuncture) was performed during the diurnal nadir (N, 0800-0900 hours) and diurnal zenith (Z, 2000-2100 hours) in the fourth (start 
a

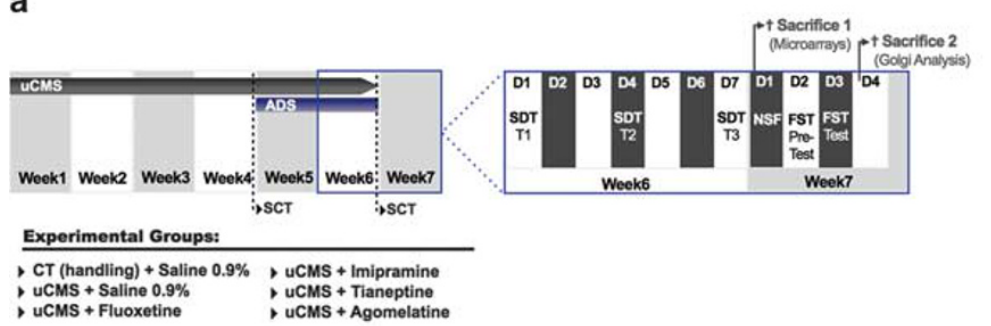

b

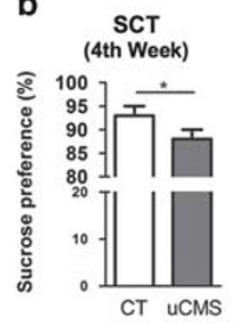

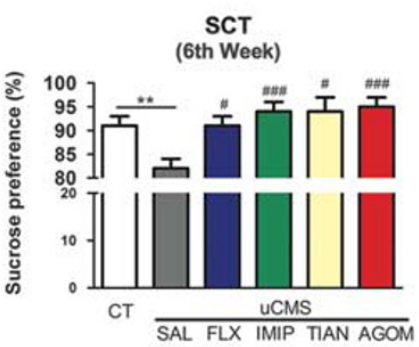

C

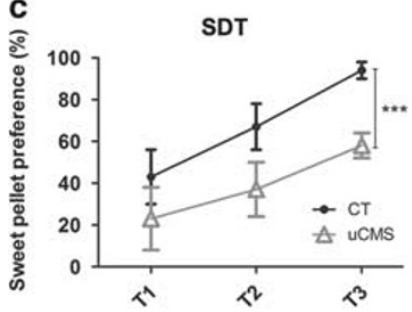

SDT-T3

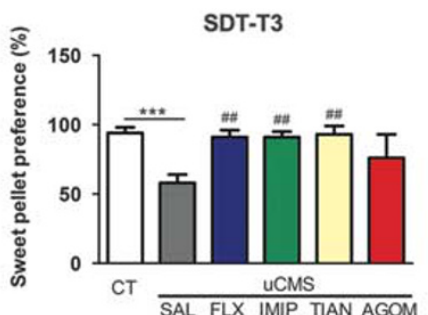

d

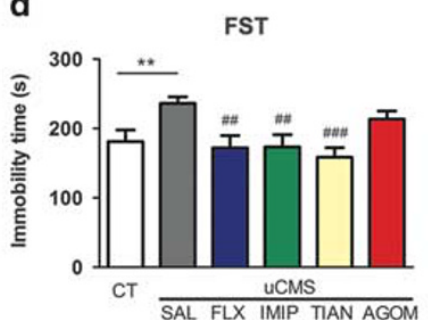

e

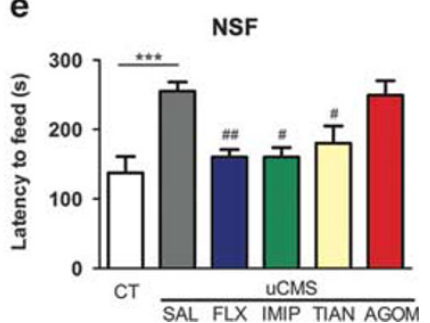

Figure I Multi-dimensional behavioral characterization of the animal model of depression used (unpredictable chronic mild stress - UCMS) before and after treatment with four different ADs. (a) UCMS protocol was applied to the animals for 6 weeks; four different ADs (FLX, fluoxetine; IMIP, imipramine; TIAN, tianeptine; AGOM, agomelatine) were administered in the last 2 weeks of the uCMS protocol. Behavioral profiling was performed using a battery of tests to assess mood and anxiety-like behavior $(n=8-12)$. Animals used for microarray analysis $(n=3)$ were killed immediately after the end of uCMS protocol/AD-treatment (Sacrifice I). Animals used for morphological analysis $(n=5 / 6)$ were killed on week 7 , after performing all behavioral tests (Sacrifice 2). (b) Sucrose consumption test (SCT) was performed at the fourth and sixth weeks of the UCMS protocol to evaluate anhedonia. uCMS induced an anhedonic profile in untreated rats (SAL), but all ADs reversed this phenotype. (c) Sweet Drive Test (SDT) was used as an additional measure of anhedonia. uCMS proved to induce anhedonia as observed in Trial 3 (T3; Trial I (TI) and Trial 2 (T2) — data not shown) that was reversed by FLX, IMIP, and TIAN but not AGOM. (d) In the Forced Swim Test (FST), uCMS induced increased immobility that was reversed by administration of FLX, IMIP, and TIAN but not AGOM. (e) UCMS exposure produced anxious-like behavior, as assessed in the Novelty Suppressed Feeding (NSF) test. All ADs, except AGOM reversed this phenotype. Error bars denote SEM. *Denotes the effect of UCMS-exposure; ${ }^{\#}$ Denotes the effect of ADs compared with untreated uCMS-exposed animals. ${ }^{* / \#} p<0.05 ;{ }^{* * / \# \#} p<0.0$ I; ${ }^{* * * * \# \#} p<0.001 . n=8-12$ animals per group.

of ADs treatment) and sixth (end of ADs treatment) weeks of the uCMS protocol.

\section{Morphological Analysis}

Three-dimensional morphometric analysis was performed on Golgi-Cox stained material obtained from rats (5-6/ group) that had been transcardially perfused with $0.9 \%$ saline and further processed, as previously described (Bessa et al, 2009a). For each animal, at least eight neurons fulfilling previously described criteria (Pinto et al, 2014) were analyzed in the dorsal and ventral hippocampal DG. For each selected neuron, dendritic branches were reconstructed at $\times 1000$ (oil) magnification using a motorized microscope (Axioplan 2; Carl Zeiss, LLC, United States) and the Neurolucida software (MBF Bioscience, Williston, VT). Three-dimensional analysis of the reconstructed neurons was performed using the NeuroExplorer software (MBF Bioscience). Measurements from individual neurons from each animal were averaged. Total dendritic length was compared among the experimental groups. Branching of the neurons was evaluated using 3D Sholl analysis; for this, the number of dendritic intersections with concentric circles positioned at radial intervals of $20 \mu \mathrm{m}$ was determined.

\section{Statistical Analysis of Behavioral Data and Biometric Parameters}

Statistical analysis was performed using the SPSS software (SPSS, Chicago, IL, USA). The underlying assumptions of all statistical procedures were assessed. The normal distribution was tested using the Kolmogorov-Smirnov test. Student's $t$-test was used to assess differences between Control and uCMS groups and one-way ANOVA followed by Bonferroni post-hoc test was performed to determine the differences between $\mathrm{AD}$-treated groups and $\mathrm{uCMS}$-exposed untreated group. Repeated-measures ANOVA was used to assess differences in the body weight changes and in the Sholl analysis of the 3D morphometric analysis. Homogeneity of variances was assessed with Levene's test when different groups were compared and with Mauchly's Test of Sphericity when repeated measures were compared. Data transformations were tested when the described assumptions were violated. As these transformations did not prove to be useful to accomplish normality or homogeneity of variances, the alternative non-parametric tests were applied (Mann-Whitney, Kruskal-Wallis, and Friedman tests). As all the significant results remained the same, the results for the parametric statistical tests were presented. Test statistics and $p$-values are shown for each test. Significance was set at $p<0.05$. Effect size, Cohen's $d$ for $t$-test (d), and eta-squared for ANOVA $\left(\eta^{2}\right)$, is presented whenever statistical significance is reached.

\section{Transcriptome Analysis}

$D G$ macrodissection. Whole DG was collected $24 \mathrm{~h}$ after the last stressor/AD injection ( $n=3$ biological replicates/group; these animals did not perform NSF and FST tests; Figure 1a). Animals were first anesthetized with pentobarbital and 
transcardially perfused with $0.9 \%$ saline. Immediately after dissection, tissues were frozen and stored at $-80{ }^{\circ} \mathrm{C}$ until further analysis.

To avoid experimenter-dependent bias, brains were macrodissected by a single investigator. Moreover, to confirm the accuracy of the macrodissections, total RNA was isolated from both the DG and the remainder hippocampus of CT animals, and gene expression analysis of genes predominantly expressed in the DG (Prox1, Dsp, and NeuroD1; Supplementary Table S1) compared with the remainder hippocampus was performed by qRT-PCR.

RNA isolation. Total RNA was isolated from the macrodissected DGs using the miRNeasy Mini kit (Qiagen, Germantown, MD, USA), according to the manufacturer's instructions. The Agilent 2100 Bioanalyzer (Agilent Technologies, Santa Clara, CA, USA) was used to assess RNA quality. Only high-quality RNA $($ RIN $>7)$ was used for microarray analysis.

Transcriptome analysis. Total RNA (200 ng) was amplified using the Ambion WT Expression Kit and the WT Terminal Labeling Kit (Affymetrix, Santa Clara, CA, USA). In all, $2.75 \mu \mathrm{g}$ of amplified cDNA was hybridized on Affymetrix Rat Gene 1.0 ST arrays containing about 28000 probe sets. Staining (Fluidics script FS450_0007) and scanning was done according to the Affymetrix expression protocol.

Expression console (v.1.2, Affymetrix) was used for quality control and to obtain annotated normalized RMA gene-level data (standard settings, including median polish and sketchquantile normalization). Statistical analyses were performed by utilizing the statistical programming environment $\mathrm{R}$ (R_Development_Core_Team, 2013) implemented in CARMAweb (Rainer et al, 2006). Genewise testing for differential expression was done by employing the limma $t$-test and Benjamini-Hochberg multiple testing correction $(\mathrm{FDR}<10 \%)$. Regulated gene sets were defined by $p<0.01$ (limma $t$-test), fold-change $>1.2 \mathrm{X}$, and an average expression $>100$ in at least one group in each pairwise comparison. Heatmaps were generated with the CARMAweb. Canonical pathway and Function and Disease enrichment analyses were done using the Ingenuity Pathway Analysis (IPA, Qiagen, Redwood City, CA, USA) software. Array data are publically accessible from NCBI/GEO (GSE56028).

Cell-type enrichment and mRNA half-life were assessed using previously published data sets (Cahoy et al, 2008; Sharova et al, 2009) and analyzed using the online resource Genes2mind (www.genes2mind.org) (Korostynski et al, 2013). Enrichment was calculated as fold difference between the level of gene expression in a particular cell type and median value of gene expression in all three cell types. Mean enrichment of gene expression was calculated as the mean value of enrichment for each set of drug-regulated genes (Cahoy et al, 2008).

cDNA synthesis and real-time PCR analysis. Total RNA $(500 \mathrm{ng})$ from the samples used for microarray analysis (DG) and from those used for determination of the macrodissections' accuracy (remainder hippocampus) was reverse-transcribed using qScript cDNA SuperMix (Quanta
Biosciences, Gaithersburg, MD, USA); for microRNAs analysis we used the qScript microRNA cDNA Synthesis Kit (qScript microRNA Quantification System, Quanta Biosciences ).

For real-time RT-PCR, oligonucleotide primers for selected genes of interest for microarrays confirmation and macrodissections' accuracy determination were designed (NCBI Primer-BLAST software) (Supplementary Table S1). Reactions were performed in an Applied Biosystems 7500 Fast Real-Time PCR System (Applied Biosystems, LLC, CA, USA) using 5X HOT FIREPol EvaGreen qPCR Mix Plus, ROX (Solis Biodyne, Tartu, Estonia), or PerfeCTa SYBR Green SuperMix, Low ROX (Quanta Biosciences) for microRNA confirmation analysis. Target gene expression levels were normalized against the housekeeping gene Beta2-Microglobulin (B2M) or RNA-U6 small nuclear 2 (RNU6) for microRNA expression analysis. The relative expression was calculated using the $\Delta \Delta \mathrm{Ct}$ method. Results are presented as fold-change of mRNA levels between the respective experimental groups after normalization to B2M or RNU6 levels.

\section{RESULTS}

\section{Multi-Dimensional Behavioral Profile}

In order to assess the common beneficial effects of four ADs belonging to different classes-fluoxetine, imipramine, tianeptine, and agomelatine, we used a well-established animal model of depression, the uCMS protocol (Figure 1a). uCMS induced anhedonia, as demonstrated by the reduced preference for a sucrose solution in the SCT, both after 4 $\left(t_{22}=1.768, p=0.045, d=0.12\right)$ and 6 weeks of uCMS exposure $\left(t_{22}=3.182, p=0.0043, d=0.3152\right.$; Figure $\left.1 b\right)$. The four ADs reversed the uCMS-induced deficits in the $\operatorname{SCT}\left(\mathrm{F}_{(4,50)}=6.468, p=0.0003, \eta^{2}=0.3410\right.$; Figure $\left.1 \mathrm{~b}\right)$. Also in the SDT, reduced preference for sweet pellets was observed throughout a three-trial testing paradigm, compared with control (CT) animals $\left(t_{19}=4.887, p=0.0001\right.$, $d=0.1884$; Figure 1c). All ADs except agomelatine reversed the uCMS anhedonic effects $\left(\mathrm{F}_{(4,43)}=5.134, p=0.0018\right.$, $\eta^{2}=0.3232$; Figure $1 \mathrm{c}$ and Supplementary Figure S1b). uCMS also induced increased immobility in the FST $\left(t_{18}=3.109, p=0.006, d=0.3493\right.$; Figure $\left.1 d\right)$, a measure of behavioral despair, which is another hallmark symptom of depressive-like behavior. Again, all ADs except agomelatine reversed this phenotype $\left(\mathrm{F}_{(4,39)}=6.197, \quad p=0.0006\right.$, $\eta^{2}=0.3886$; Figure 1d).

Anxious-like behavior was assessed in the NSF test (Figure 1e and Supplementary Figure S1c). As expected, uCMS-exposed animals presented higher latency to feed in the NSF compared with CT $\left(t_{22}=4.303, p=0.0003\right.$, $d=0.4570$; Figure $1 \mathrm{e})$, indicating increased anxietylike phenotype. All ADs except agomelatine reversed this phenotype $\left(\mathrm{F}_{(4,55)}=7.335, \quad p<0.0001, \quad \eta^{2}=0.3479\right.$; Figure 1e).

As an additional measure of face and predictive validity, corticosterone levels were measured in the serum (Supplementary Figure S2). Disruption of the diurnal pattern of corticosterone production in uCMS-exposed animals and reversion by ADs was observed (Supplementary Figure S2). 


\section{Neuroplastic Changes in the Hippocampal DG}

Neuroplastic changes were assessed in the dorsal (dDG) and ventral (vDG) hippocampal DG. As previously described (Bessa et al, 2009a; Pinto et al, 2014), uCMS-exposed animals presented significantly shorter granule neurons in the dDG compared with CT animals $\left(t_{8}=4.560, p=0.0019\right.$, $d=0.7221$; Figure 2a). All ADs, excluding agomelatine, reversed this structural change up to the levels of CT $\left(\mathrm{F}_{(4,21)}=5.344, p=0.0040, \eta^{2}=0.5054\right.$; Figure 2a). Sholl analysis revealed less complex granule neurons in uCMSexposed animals compared with CT and a reversion by tianeptine and imipramine (Supplementary Figure S3a).

Concerning the vDG, no statistically significant differences were observed in the dendritic length between the experimental groups (CT $v s$ uCMS: $t_{11}=0.7387, p=0.4756$; uCMS_ADs $v s$ uCMS: $\mathrm{F}_{(4,21)}=1.910, p=0.1463$; Figure $\left.2 \mathrm{~b}\right)$. Interestingly, however, the Sholl analysis disclosed significantly more complex neurons in the uCMS animals compared with fluoxetine and agomelatine-treated animals (Supplementary Figure S3b).

\section{Microarray Analysis of the DG of uCMS-Exposed and AD-Treated Rats}

To unravel the molecular regulation in the hippocampal DG after uCMS exposure and treatment with each of the four ADs, microarray analysis of macrodissected DG was performed. To confirm the specificity of the macrodissections, gene expression analysis was performed in the DG of $\mathrm{CT}$ animals and compared with the remainder tissue of the hippocampus. The accuracy of the dissections was validated by an enrichment of genes predominantly expressed in the DG (Neurogenic differentiation 1 (NeuroD1) Desmoplakin, (Dsp) and Prospero homeobox 1 (Prox1)) compared with the remaining hippocampus (Supplementary Figure S4). A heatmap and a cluster dendrogram were generated according to the expression profile of 1311 significantly regulated $(p<0.01)$ probe sets (Figure 2c). Mild gene expression changes were observed, with most transcripts presenting fold changes between 1.2 and 1.5 (Figure 2d). This genome-wide analysis revealed both upregulation and downregulation of a large number of molecules, mainly in
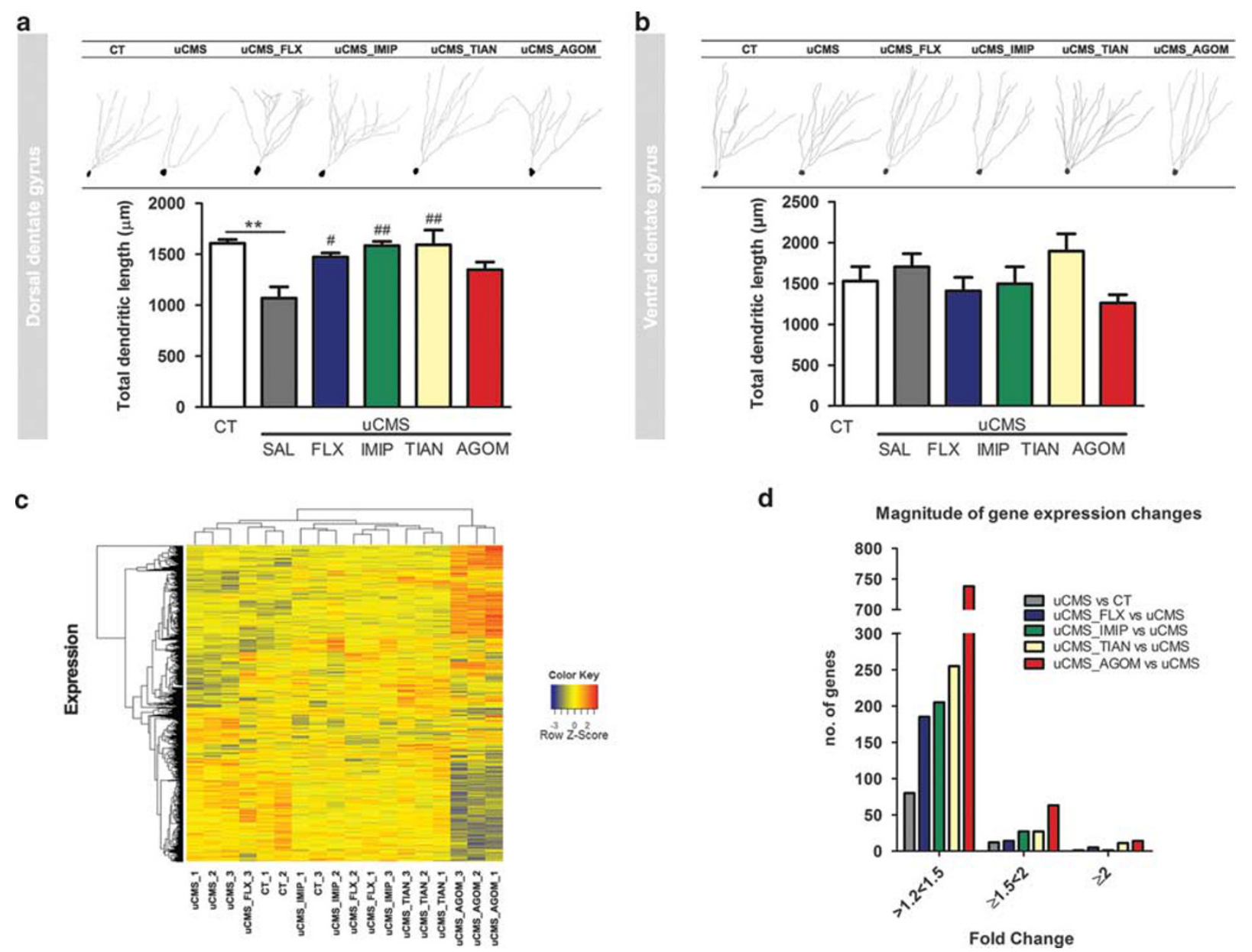

Figure 2 Neuronal morphology and gene expression analysis of the hippocampal DG. (a) Representative pictures of reconstructed Golgi-impregnated granule neurons of the dorsal DG and average total dendritic length. Untreated uCMS-exposed animals (SAL) show a decrease in the total dendritic length that is recovered by fluoxetine (FLX), imipramine (IMIP), and tianeptine (TIAN) administration. (b) Representative pictures of reconstructed Golgiimpregnated granule neurons of the ventral DG and total dendritic length. No statistically significant differences were observed between groups. Error bars denote SEM. * Denotes the effect of uCMS-exposure; \#Denotes the effect of antidepressants compared with untreated uCMS-exposed animals.

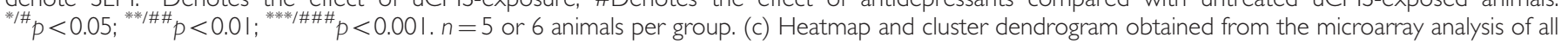
samples generated according to the expression profile of I 3 I I significantly regulated $(p<0.0 \mathrm{I})$ probe sets. (d) Magnitude of the transcriptional response to chronic stress exposure (UCMS) and ADs' treatment in the DG; the majority of fold changes are between 1.2- and I.5-fold. 
AD-treated animals (Figure 3a). The ratio between upregulated and downregulated genes was positive for all ADs, except agomelatine (FLX: 2.2; IMIP: 1.8; TIAN: 5; AGOM: $0.89)$.

\section{Transcriptional Regulation Induced by uCMS Exposure} and $\mathrm{AD}$ Treatment

A total of 93 transcripts were altered in uCMS, when compared with CTs (Figure 3a). The top 10 regulated functional terms associated with these probe sets were identified (Figure 3b). Functions related to the categories Cancer, Inflammatory Disease, and Metabolic Disease showed the highest number of altered transcripts.

Regarding the effects of AD administration, a total of 209 transcripts in fluoxetine-treated animals, 293 in tianeptinetreated animals, 233 in imipramine-treated animals, and 815 in agomelatine-treated animals were regulated compared with uCMS animals (Figure 3a and $\mathrm{c}$ and Supplementary Table S2). Despite the higher number of regulated transcripts in agomelatine-treated animals, fluoxetine, imipramine, and tianeptine reversed more uCMS-induced changes, thus normalizing the levels of these molecules to the levels of CT $(25,22$, and $32 \%$ of reversion, respectively, compared with $12 \%$ in agomelatine-treated animals; Table 1 and Supplementary Table S3). Of notice, the list of genes whose expression was reversed after AD treatment included several microRNAs (eg, miR-409, miR-411), dual specificity protein phosphatase 1 (Dusp1), and Metallothionein 4 (Mt4) (Table 1).

\section{ADs Commonly Regulated Genes}

Independently of the $\mathrm{AD}$ treatment, 11 transcripts were commonly regulated (Figure 3c). The majority of them are novel transcripts or coding for uncharacterized predicted proteins (Supplementary Table S4). As most of the behavioral effects were observed after treatment with fluoxetine, imipramine, or tianeptine, and because these three ADs could reverse more transcriptional alterations induced by uCMS, we decided to further explore their common effects. The analysis of commonly regulated genes revealed 33 probe sets, including several small nucleolar RNAs (SnoRNA) and microRNAs (eg, miR-409, miR-411, miR-412), tissue plasminogen activator (Plat), Mt4, and Dusp1 (Supplementary Tables S4 and S5). A predicted network where these genes interact to induce the cellular and behavioral beneficial effects observed is depicted in Supplementary Figure S5. The IPA software functions associated to this network are related to tissue morphology and connective tissue development and function.

To confirm the differential expression of genes identified by microarray analysis, real-time RT-PCR of the selected genes for each relevant comparison was performed (Figure $3 \mathrm{~d}-\mathrm{h}$ ). These genes were chosen based on their neuroplasticity-related function; those without neuroplasticity-related function were randomly selected from the list of significantly altered genes to avoid bias in the microarray confirmation. Moreover, genes with annotated function whose expression was reversed by at least three of the a

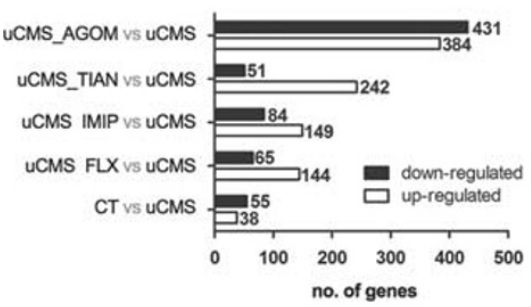

d

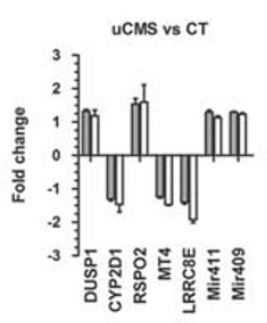

e

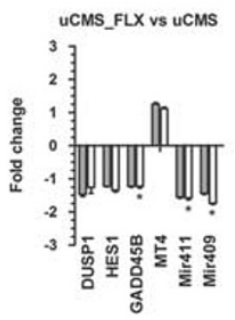

b

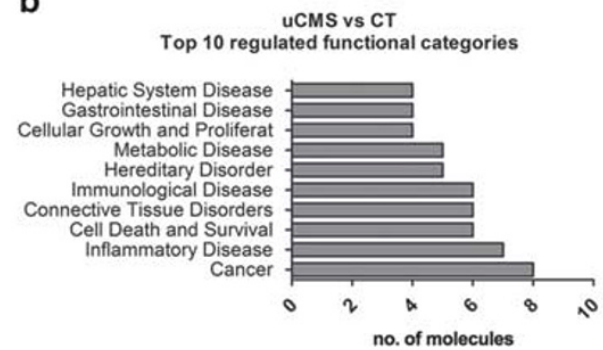

f

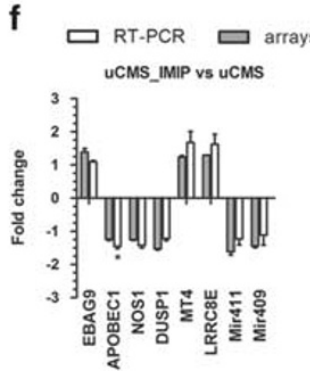

g

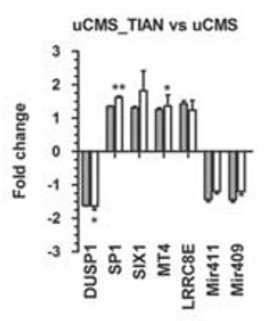

C

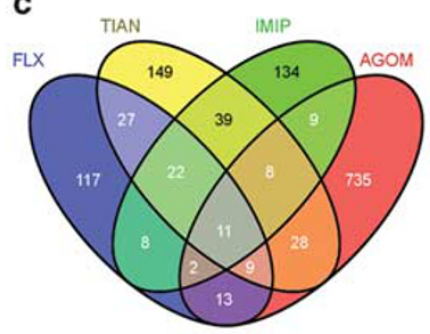

h

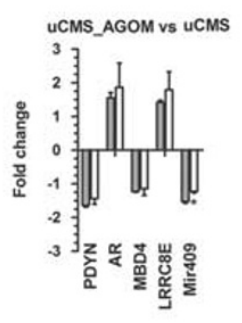

i

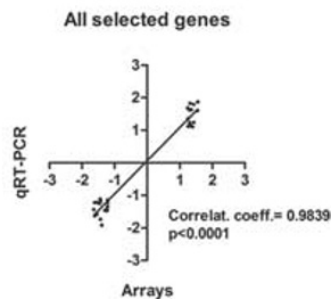

Figure 3 Microarray analysis of the DG of Control, uCMS-exposed and antidepressant-treated rats. (a) The number of upregulated and downregulated genes in UCMS vs CT animals and in AD-treated vs UCMS animals. (b) Top I0 significantly enriched functional categories identified with Ingenuity Pathway Analysis (IPA) Software in uCMS-exposed vs Control animals. Shown are the terms with the highest number of genes, as indicated by the bars. (c) Venn diagram depicting the number of significantly regulated transcripts $(p<0.01$, fold change $(F C)>1.2 \times$, average expression $>100)$ after 2 weeks of $A D$ treatment and overlap between treatments. ( $d-h$ ) Confirmation of the microarrays data was performed by qRT-PCR of selected genes, for each relevant comparison. White bars depict the linear FC of mRNA levels between the respective experimental groups after normalization to B2M/RNU6 (microRNAs) mRNA levels; Grey bars depict the observed linear FC in the Affymetrix microarrays. Error bars denote SEM. *Denotes statistical differences between groups for each relevant comparison after normalization to B2M/RNU6 mRNA levels; * $p<0.05 ; n=3$ per group. (i) Linear regression analysis of FC from microarrays and qRT-PCR of all selected genes for confirmation. Correlation coefficient and p-value are indicated in the graph. AGOM, agomelatine; $\mathrm{CT}$, control; FLX, fluoxetine; IMIP, imipramine; TIAN, tianeptine; UCMS, unpredictable chronic mild stress. 
Molecular regulation in the dentate gyrus by antidepressants

P Patrício et al

Table I List of uCMS-Induced Transcriptional Alterations Reversed by Antidepressants (ADs) Treatment

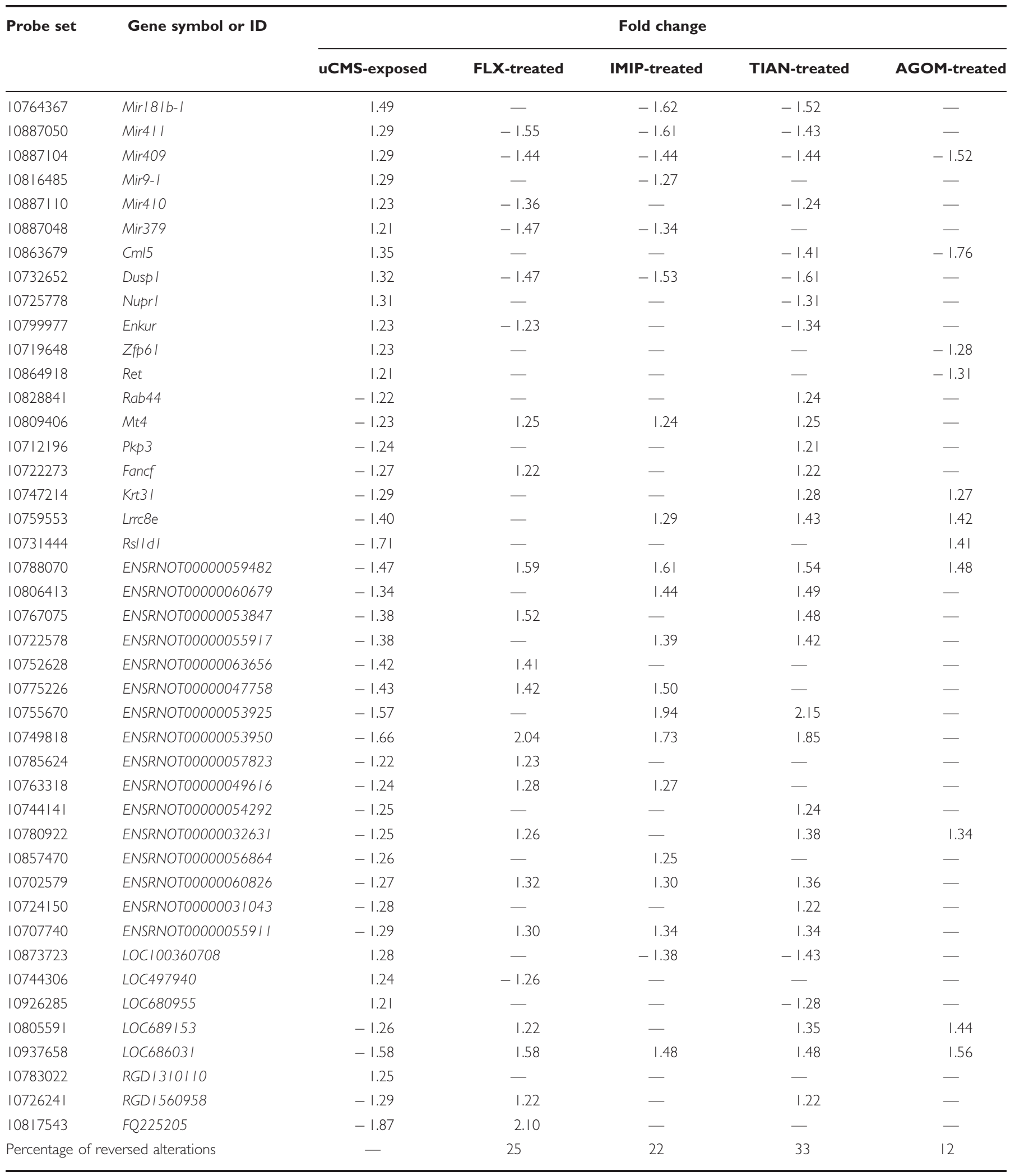

Last row shows the percentage of molecules whose expression was reversed by each of the ADs.

ADs were analyzed (Supplementary Table S1). For all the selected genes, the differential expression observed by Affymetrix arrays (Figure $3 \mathrm{~d}-\mathrm{h}$ ) was confirmed. Moreover, a strong correlation between the microarrays and the qRTPCR fold changes was found (correlation coefficient 0.9839 , $p<0.0001$; Figure 3i). 


\section{Canonical Pathways Analysis}

PA software was used to identify pathways sensitive to stress and to AD treatment. A list of significantly enriched canonical pathways and the corresponding genes is provided for each relevant comparison (Figure 4a-d and Supplementary Table S6). Chronic stress exposure induced the upregulation of only one gene related to the glutathione redox reactions pathway. Fluoxetine treatment promoted, among others, the downregulation of genes involved in pro-inflammatory response pathways (eg, IL-6 signaling, NF- $\kappa \beta$ signaling, acute phase response signaling; Figure $4 \mathrm{a}$ ) and the upregulation of genes from metabolic pathways (eg, pentose phosphate pathway and PPAR signaling; Figure 4a). Imipramine treatment induced the downregulation of several genes involved in drug metabolism pathways and the upregulation of DNA-damage and oxidative stress response pathwaysrelated genes (eg, DNA double-strand break repair by nonhomologous end joining, NRF2-mediated oxidative stress response; Figure $4 \mathrm{~b}$ ). Tianeptine treatment also promoted the downregulation of genes from drug metabolism pathways and the upregulation of biosynthetic and DNA-damage response pathways (Figure 4c). Finally, agomelatine treatment induced the downregulation of genes involved in Cdk5 Signaling, Netrin signaling, and synaptic long-term depression and the upregulation of genes involved in RhoGTPases-related pathways (Figure $4 \mathrm{~d}$ and Supplementary Table S6).

\section{Nervous Tissue Cell-Type Enrichment and Transcript Stability Analysis}

To understand the cellular impact of each $\mathrm{AD}$, we analyzed which cells types were expressing the genes significantly altered after uCMS exposure and AD treatment. For that, publicly available data representing cellular enrichment of individual transcripts in neurons, astrocytes, or oligodendrocytes were used (Cahoy et al, 2008; Korostynski et al, 2013; Figure 4e; and Supplementary Figure S6). Fluoxetine treatment was characterized by an enrichment of genes expressed in neurons, whereas agomelatine treatment showed an enrichment of genes expressed both in oligodendrocytes and neurons, in comparison to astrocytes. Imipramine and a

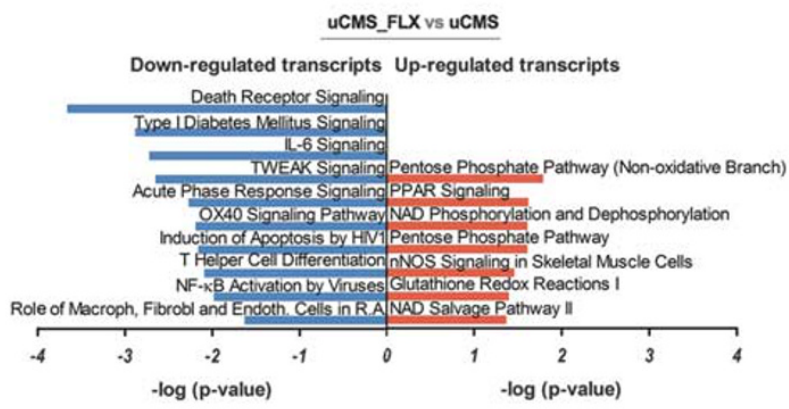

C

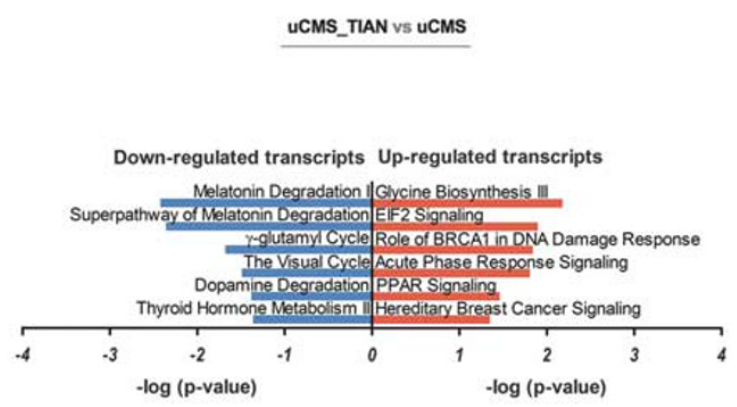

b

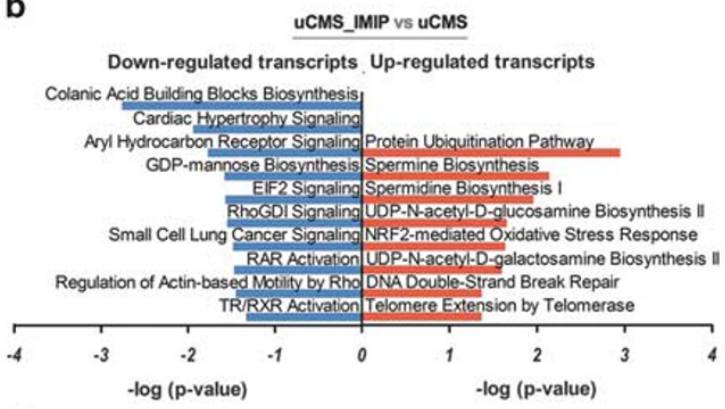

d

e
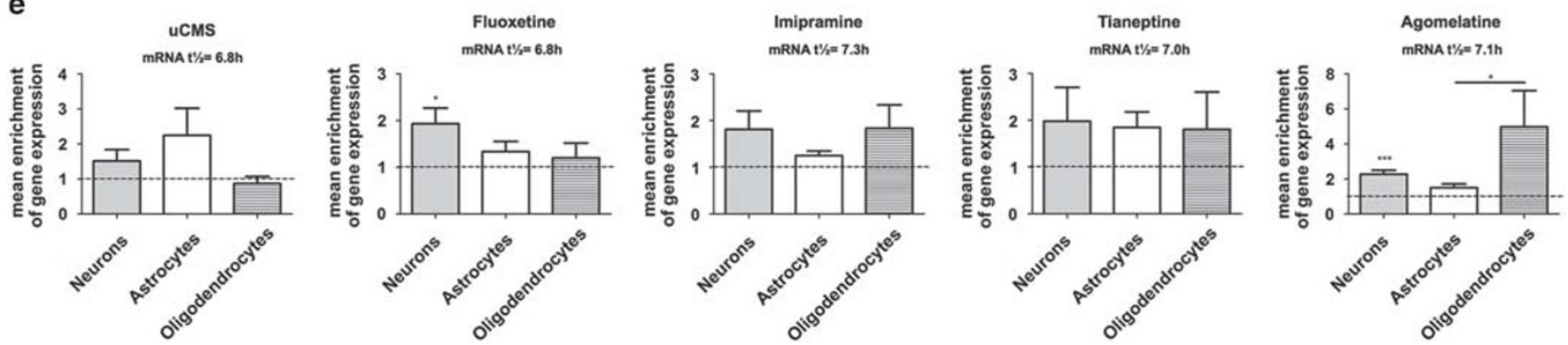

Figure 4 Characterization of ADs' effects and cellular targets. (a-d) Top 10 significantly enriched canonical pathways identified with Ingenuity Pathway Analysis (IPA) Software in untreated UCMS rats vs FLX-treated (a), IMIP-treated (b), TIAN-treated (c), and AGOM-treated (d) animals. Pathways presenting the higher number of regulated transcripts were selected. (e) Predicted cell type enrichment and median mRNA half-life (mRNA t1/2) of uCMS- and ADsresponsive genes. Median mRNA t1/2 for whole transcriptome was 7.I h. Error bars denote SEM. * $p<0.05$; **** $p<0.00$ I. AGOM, agomelatine; CT, control; FLX, fluoxetine; IMIP, imipramine; TIAN, tianeptine; uCMS, unpredictable chronic mild stress. 
tianeptine treatments did not reveal enrichment in any particular cell type. Although not reaching statistical significance, uCMS exposure showed a trend for enrichment in transcripts from astrocytes and neurons compared with oligodendrocytes (Figure 4e and Supplementary Figure S6).

To further dissect the function of the differentially regulated genes, we analyzed their transcript stability (Schwanhausser et al, 2011). Genes with low mRNA stability are frequently involved in the regulation of intracellular signaling, whereas long-lived transcripts have a role in cell metabolism (Korostynski et al, 2013; Schwanhausser et al, 2011). Similar median mRNA half-life for each of the treatments was found (Figure $4 \mathrm{e}$ ). Moreover, these values were close to the whole genome median mRNA half-life $(7.1 \mathrm{~h})$.

\section{DISCUSSION}

Although most ADs produce similar behavioral and neuroplastic effects, each AD has a characteristic pharmacological and molecular signature, the full exploitation of which could be helpful in designing treatments that capture the various pathological facets presented by individual depressed patients. To this end, we here sought to identify common and divergent molecular targets and pathways of four distinct classes of ADs, represented by fluoxetine, imipramine, tianeptine, and agomelatine. Our genome-wide analysis focused on the hippocampal DG-one of the most studied neural targets of stress and ADs (Lucassen et al, 2014; Wainwright and Galea, 2013)_from animals displaying behavioral and endocrine impairments akin to depression. These behavioral anomalies were reversed after 2 weeks of treatment with fluoxetine, imipramine, and tianeptine; agomelatine resulted in only partial behavioral recovery although it re-synchronized the diurnal pattern of corticosterone secretion. Notably, fluoxetine, imipramine, and tianeptine, but not agomelatine, also restored dendritic arborization of the dorsal dentate granule cells of the hippocampus to prestress levels. No differences were disclosed in neuronal dendritic length in the vDG. Given the evidence for a heterogeneous structure and function along the septotemporal axis of the DG (Kheirbek et al, 2013; Tanti and Belzung, 2013), with the dDG contributing mainly to functions related to learning and memory, whereas the vDG is more related to anxiety and emotional regulation (Tanti and Belzung 2013), we would expect also an impact on the vDG. Moreover, while previous reports have shown that the effects of some AD treatments on adult hippocampal neurogenesis are region specific along the septo-temporal axis of the hippocampus, the implication of morphological changes in granule neurons has not been fully elucidated (Felice et al, 2012; O'Leary et al, 2012), even though a contrasting gradient of stress-induced morphological and physiological changes along the septo-temporal axis of the hippocampus has been shown (Pinto et al, 2014).

\section{Transcriptional Changes Induced by uCMS and Common Reversal Mechanisms by AD Treatment}

Depression is associated with risk for other pathologies, including cancer and cardiometabolic disease (Lang and Borgwardt, 2013), which themselves are associated with stress. The present analysis of uCMS-exposed animals revealed upregulation and downregulation of genes related to these disorders.

Dusp1, a key negative regulator of the MAP kinase signaling pathway (Huang and Tan, 2012), previously related to the pathophysiology of depression in humans and animal models of depression (Duric et al, 2010) was upregulated by uCMS exposure. Importantly, MAP kinase pathway has been previously implicated in synaptic plasticity (Duric et al, 2010; Sweatt, 2004) and may partly explain the altered neuroplasticity observed in the dDG of uCMS-exposed animals. Consistent with their behavior- and neuroplastic-improving actions, all ADs except agomelatine, reversed uCMS-upregulated Dusp1 expression. Interestingly, at least two other studies have shown the involvement of MAP kinase pathway-related genes in the actions of monoaminergic ADs (Malki et al, 2012; Surget et al, 2009), further endorsing the role of this pathway as a common strategy used by different $\mathrm{AD}$ treatments to reverse the depressive-like behaviors. We also observed that a significant number of miRNA precursors were upregulated and downregulated by uCMS and ADs, respectively. Importantly, among the predicted targets of these miRNAs are genes of the MAP kinase pathway (eg, Map2k1; Map3k1), calcium-signaling-related genes (eg, Calml4, Camkk2), and Rho-signaling-related genes (eg, RhoGef, Rnd2) (Lewis et al, 2005). This finding is interesting in light of the role of miRNAs in neuronal development and neuroplasticity (McClung and Nestler, 2008) and their potential for serving as new therapeutic targets (Hansen and Obrietan, 2013). Indeed, several recent studies have demonstrated that miRNAs are both targets not only for disruption in mental illness (Kohen et al, 2014) but also for $\mathrm{AD}$ treatment action (Baudry et al, 2010; O'Connor et al, 2013).

Noticeably, and given the role of the hippocampal DG cell genesis changes in the onset of depressive-like behavior and in the actions of ADs, only minor contribution of key neurogenesis-related genes emerged from the comparisons between experimental groups (eg, Sox11, Hes1). We assume that this under-representation may be due to the small proportion of progenitor cells in the tissue (representative of the SGZ) when compared with the remainder mature cells. However, an enrichment of genes related to particular aspects of neurogenesis, such as neuronal migration/ axonogenesis (eg, RhoC, Slit1, Epha6, Rasa1, Mapk3, Cxcl12) and neuronal fate determination and plasticity (eg, Dusp1, Cdc20), was found.

\section{AD-specific Transcriptional Changes}

The present transcriptome analysis showed that not only each class of $\mathrm{AD}$ left its own molecular signature on the DG but also that they triggered common regulatory effects on a number of gene families and pathways.

Depressed patients and animal models of depression frequently display a deregulated neuroinflammatory response (Shelton et al, 2011; Sukoff Rizzo et al, 2012), resulting in increased production of pro-inflammatory cytokines that alter neurotransmitter metabolism and neural plasticity (Willner et al, 2013). It was interesting to observe here that fluoxetine reduced the expression of IL6-signaling and of 
TNF signaling-related molecules. These pathways have been implicated in the development of depressive-like behaviors (Manosso et al, 2013) and were shown to be overrepresented in this and other brain regions in response to chronic stress (Datson et al, 2012; Sukoff Rizzo et al, 2012). In addition, fluoxetine treatment in uCMS rats activated pathways related to cellular respiration and metabolism, a finding in line with the presence of long-lived transcripts (Korostynski et al, 2013; Schwanhausser et al, 2011). Further, consistent with the findings of other studies (Encinas et al, 2006; Mateus-Pinheiro et al, 2013b; Surget et al, 2011), the actions of fluoxetine were more pronounced in neurons than in astrocytes and oligodendrocytes.

Interestingly, imipramine and tianeptine were found to act similarly; the mechanism of action of tianeptine, a drug structurally similar to tricyclic agents, is largely unknown (McEwen et al, 2010). However, we recently predicted that the transcriptional effects of tianeptine, like those of imipramine, may involve the modulation of norepinephrine, serotonin, and dopamine systems in the striatum of naive mice (Korostynski et al, 2013). In the present study, imipramine and tianeptine co-regulated a large number of genes; notably, those were implicated in pathways related to drug metabolism, biosynthesis, and DNA-damage response; this finding suggests that the mechanisms of action of the two drugs may involve neuroprotection against the neurotoxic effects of stress. Moreover, as both neuronal and non-neuronal cells were predicted to respond to these drugs, it is likely that they exert broad actions that ultimately converge to reverse the harmful structural and molecular effects of uCMS.

In contrast to imipramine, fluoxetine, and tianeptine, agomelatine acts on two pharmacological substrates: melatonin and $5 \mathrm{HT}_{2 \mathrm{c}}$ receptors. Thus, it was not surprising that its application produced a behavioral and molecular therapeutic profile that was distinct from that of the other ADs tested. Remarkably, agomelatine produced the highest number of transcriptional changes but reversed only $12 \%$ of the transcriptional changes induced by uCMS. Rho-GTPasesignaling related genes were among those showing the highest upregulation by agomelatine. Although this signaling pathway is an important regulator of morphological neuroplasticity (Negishi and Katoh, 2005), agomelatine was strikingly poor in reversing the maladaptive structural (and behavioral) alterations induced by uCMS. In silico analysis predicted that agomelatine acts on neurons and oligodendrocytes, matching previous observations that melatonin promotes oligodendroglial maturation (Olivier et al, 2009).

This study identifies new molecular correlates of chronic stress that are subject to differential regulation by different classes of ADs and which may therefore underlie their different efficacies in reversing the maladaptive neurostructural and behavioral changes observed in the DG of animal models of depression. Interestingly, and according to previous studies (Datson et al, 2012), little overlap was found between the $\mathrm{AD}$-regulated genes and pathways in the context of this paradigm of induced depressive-like behavior ( $\mathrm{uCMS}$ ) and those regulated in naive $\mathrm{AD}$-treated animals (Gaska et al, 2012; Landgrebe et al, 2002; Sillaber et al, 2008). This further emphasizes the relevance of using animal models of depression to explore the molecular mechanisms of depressive-like phenotype reversion in the brain. As a result, the new information gained may allow exploitation of the unique properties of each $\mathrm{AD}$ in the search for the next generation of ADs; to further pursue this aim, molecular profiling of other brain regions affected in depression will be necessary. The data reported here may also serve to guide drug choice in managing symptoms in individual patients with specific genetic variants.

\section{FUNDING AND DISCLOSURE}

Patrícia Patrício, António Mateus-Pinheiro, Mónica Morais, and Nuno Dinis Alves received fellowships from the Portuguese Foundation for Science and Technology (FCT). Michal Korostynski and Marcin Piechota were funded by the POIG De-Me-Ter 3.1 and NCN 2011/03/D/NZ3/01686 grants. This study was co-funded by the Life and Health Sciences Research Institute (ICVS) and ON.2-O NOVO NORTE-North Portugal Regional Operational Programme 2007/2013, of the National Strategic Reference Framework (NSRF) 2007/2013, through the European Regional Development Fund (ERDF) and by the SwitchBox Consortium (Contract FP7-Health-F2-2010-259772 from the European Union). The authors declare no conflict of interest.

\section{ACKNOWLEDGEMENTS}

We thank Luís Martins, Miguel Carneiro, Ana Lima and Anke Bettenbrock for excellent technical assistance. We also thank Patrício Costa for advice on statistical analysis.

\section{REFERENCES}

Andrus BM, Blizinsky K, Vedell PT, Dennis K, Shukla PK, Schaffer DJ et al (2012). Gene expression patterns in the hippocampus and amygdala of endogenous depression and chronic stress models. Mol Psychiatry 17: 49-61.

Banasr M, Soumier A, Hery M, Mocaer E, Daszuta A (2006). Agomelatine, a new antidepressant, induces regional changes in hippocampal neurogenesis. Biol Psychiatry 59: 1087-1096.

Baudry A, Mouillet-Richard S, Schneider B, Launay JM, Kellermann O (2010). miR-16 targets the serotonin transporter: a new facet for adaptive responses to antidepressants. Science 329: 1537-1541.

Bessa JM, Ferreira D, Melo I, Marques F, Cerqueira JJ, Palha JA et al (2009a). The mood-improving actions of antidepressants do not depend on neurogenesis but are associated with neuronal remodeling. Mol Psychiatry 14: 764-773, 739.

Bessa JM, Mesquita AR, Oliveira M, Pego JM, Cerqueira JJ, Palha JA et al (2009b). A trans-dimensional approach to the behavioral aspects of depression. Front Behav Neurosci 3: 1.

Bessa JM, Morais M, Marques F, Pinto L, Palha JA, Almeida OF et al (2013). Stress-induced anhedonia is associated with hypertrophy of medium spiny neurons of the nucleus accumbens. Transl Psychiatry 3: e266.

Blier P, Blondeau C (2011). Neurobiological bases and clinical aspects of the use of aripiprazole in treatment-resistant major depressive disorder. J Affect Disord 128(Suppl 1): S3-S10.

Cahoy JD, Emery B, Kaushal A, Foo LC, Zamanian JL, Christopherson KS et al (2008). A transcriptome database for astrocytes, neurons, and oligodendrocytes: a new resource for understanding brain development and function. J Neurosci 28: 264-278.

Conti B, Maier R, Barr AM, Morale MC, Lu X, Sanna PP et al (2007). Region-specific transcriptional changes following the three antidepressant treatments electro convulsive therapy, sleep deprivation and fluoxetine. Mol Psychiatry 12: 167-189. 
Cryan JF, Slattery DA (2007). Animal models of mood disorders: recent developments. Curr Opin Psychiatry 20: 1-7.

Datson NA, Speksnijder N, Mayer JL, Steenbergen PJ, Korobko O, Goeman J et al (2012). The transcriptional response to chronic stress and glucocorticoid receptor blockade in the hippocampal dentate gyrus. Hippocampus 22: 359-371.

Drigues N, Poltyrev T, Bejar C, Weinstock M, Youdim MB (2003). cDNA gene expression profile of rat hippocampus after chronic treatment with antidepressant drugs. J Neural Transm 110: 1413-1436.

Duric V, Banasr M, Licznerski P, Schmidt HD, Stockmeier CA, Simen AA et al (2010). A negative regulator of MAP kinase causes depressive behavior. Nat Med 16: 1328-1332.

Encinas JM, Vaahtokari A, Enikolopov G (2006). Fluoxetine targets early progenitor cells in the adult brain. Proc Natl Acad Sci USA 103: $8233-8238$.

Felice D, O’Leary OF, Pizzo RC, Cryan JF (2012). Blockade of the GABA(B) receptor increases neurogenesis in the ventral but not dorsal adult hippocampus: relevance to antidepressant action. Neuropharmacology 63: 1380-1388.

Gaska M, Kusmider M, Solich J, Faron-Gorecka A, Krawczyk MJ, Kulakowski K et al (2012). Analysis of region-specific changes in gene expression upon treatment with citalopram and desipramine reveals temporal dynamics in response to antidepressant drugs at the transcriptome level. Psychopharmacology (Berl) 223: 281-297.

Hansen KF, Obrietan K (2013). MicroRNA as therapeutic targets for treatment of depression. Neuropsychiatr Dis Treat 9: 1011-1021.

Hill MN, Hellemans KG, Verma P, Gorzalka BB, Weinberg J (2012). Neurobiology of chronic mild stress: parallels to major depression. Neurosci Biobehav Rev 36: 2085-2117.

Huang CY, Tan TH (2012). DUSPs, to MAP kinases and beyond. Cell Biosci 2: 24.

Kheirbek MA, Drew LJ, Burghardt NS, Costantini DO, Tannenholz L, Ahmari SE et al (2013). Differential control of learning and anxiety along the dorsoventral axis of the dentate gyrus. Neuron 77: 955-968.

Kohen R, Dobra A, Tracy JH, Haugen E (2014). Transcriptome profiling of human hippocampus dentate gyrus granule cells in mental illness. Transl Psychiatry 4: e366.

Korostynski M, Piechota M, Dzbek J, Mlynarski W, Szklarczyk K, Ziolkowska B et al (2013). Novel drug-regulated transcriptional networks in brain reveal pharmacological properties of psychotropic drugs. BMC Genomics 14: 606.

Landgrebe J, Welzl G, Metz T, van Gaalen MM, Ropers H, Wurst W et al (2002). Molecular characterisation of antidepressant effects in the mouse brain using gene expression profiling. J Psychiatr Res 36: 119-129.

Lang UE, Borgwardt S (2013). Molecular mechanisms of depression: perspectives on new treatment strategies. Cell Physiol Biochem 31: 761-777.

Lee JH, Ko E, Kim YE, Min JY, Liu J, Kim Y et al (2010). Gene expression profile analysis of genes in rat hippocampus from antidepressant treated rats using DNA microarray. $B M C$ Neurosci 11: 152.

Lewis BP, Burge CB, Bartel DP (2005). Conserved seed pairing, often flanked by adenosines, indicates that thousands of human genes are microRNA targets. Cell 120: 15-20.

Li X, Frye MA, Shelton RC (2012). Review of pharmacological treatment in mood disorders and future directions for drug development. Neuropsychopharmacology 37: 77-101.

Lisowski P, Juszczak GR, Goscik J, Stankiewicz AM, Wieczorek M, Zwierzchowski L et al (2013). Stress susceptibility-specific phenotype associated with different hippocampal transcriptomic responses to chronic tricyclic antidepressant treatment in mice. BMC Neurosci 14: 144.

Lucassen PJ, Pruessner J, Sousa N, Almeida OF, Van Dam AM, Rajkowska G et al (2014). Neuropathology of stress. Acta Neuropathol 127: 109-135.
Malki K, Lourdusamy A, Binder E, Paya-Cano J, Sluyter F, Craig I et al (2012). Antidepressant-dependent mRNA changes in mouse associated with hippocampal neurogenesis in a mouse model of depression. Pharmacogenet Genomics 22: 765-776.

Manosso LM, Neis VB, Moretti M, Daufenbach JF, Freitas AE, Colla AR et al (2013). Antidepressant-like effect of alpha-tocopherol in a mouse model of depressive-like behavior induced by TNFalpha. Prog Neuropsychopharmacol Biol Psychiatry 46: 48-57.

Mateus-Pinheiro A, Patricio P, Alves ND, Machado-Santos AR, Morais M, Bessa JM et al (2014). The Sweet Drive Test: refining phenotypic characterization of anhedonic behavior in rodents. Front Behav Neurosci 8: 74 .

Mateus-Pinheiro A, Patricio P, Bessa JM, Sousa N, Pinto L (2013a). Cell genesis and dendritic plasticity: a neuroplastic pas de deux in the onset and remission from depression. Mol Psychiatry 18: 748-750.

Mateus-Pinheiro A, Pinto L, Bessa JM, Morais M, Alves ND, Monteiro S et al (2013b). Sustained remission from depressive-like behavior depends on hippocampal neurogenesis. Transl Psychiatry 3: e210.

McClung CA, Nestler EJ (2008). Neuroplasticity mediated by altered gene expression. Neuropsychopharmacology 33: 3-17.

McEwen BS, Chattarji S, Diamond DM, Jay TM, Reagan LP, Svenningsson $\mathrm{P}$ et al (2010). The neurobiological properties of tianeptine (Stablon): from monoamine hypothesis to glutamatergic modulation. Mol Psychiatry 15: 237-249.

Nakatani N, Aburatani H, Nishimura K, Semba J, Yoshikawa T (2004). Comprehensive expression analysis of a rat depression model. Pharmacogenomics J 4: 114-126.

Negishi M, Katoh H (2005). Rho family GTPases and dendrite plasticity. Neuroscientist 11: 187-191.

O'Connor RM, Grenham S, Dinan TG, Cryan JF (2013). microRNAs as novel antidepressant targets: converging effects of ketamine and electroconvulsive shock therapy in the rat hippocampus. Int J Neuropsychopharmacol 16: 1885-1892.

O'Leary OF, O'Connor RM, Cryan JF (2012). Lithium-induced effects on adult hippocampal neurogenesis are topographically segregated along the dorso-ventral axis of stressed mice. Neuropharmacology 62: 247-255.

Olivier P, Fontaine RH, Loron G, Van Steenwinckel J, Biran V, Massonneau V et al (2009). Melatonin promotes oligodendroglial maturation of injured white matter in neonatal rats. PLos One 4: e7128.

Pinto V, Costa JC, Morgado P, Mota C, Miranda A, Bravo FV et al (2014). Differential impact of chronic stress along the hippocampal dorsal-ventral axis. Brain Struct Funct, (e-pub ahead of print 6 February 2014; doi:10.1007/s00429-014-0713-0).

Pittenger C, Duman RS (2008). Stress, depression, and neuroplasticity: a convergence of mechanisms. Neuropsychopharmacology 33: 88-109.

R_Development_Core_Team (2013): R: A language and environment for statistical computing. http://www.R-project.org/.

Rainer J, Sanchez-Cabo F, Stocker G, Sturn A, Trajanoski Z (2006). CARMAweb: comprehensive R- and bioconductor-based web service for microarray data analysis. Nucleic Acids Res 34(Web Server issue): W498-W503.

Schwanhausser B, Busse D, Li N, Dittmar G, Schuchhardt J, Wolf J et al (2011). Global quantification of mammalian gene expression control. Nature 473: 337-342.

Sharova LV, Sharov AA, Nedorezov T, Piao Y, Shaik N, Ko MS (2009). Database for mRNA half-life of 19977 genes obtained by DNA microarray analysis of pluripotent and differentiating mouse embryonic stem cells. DNA Res 16: 45-58.

Shelton RC, Claiborne J, Sidoryk-Wegrzynowicz M, Reddy R, Aschner M, Lewis DA et al (2011). Altered expression of genes involved in inflammation and apoptosis in frontal cortex in major depression. Mol Psychiatry 16: 751-762.

Sillaber I, Panhuysen M, Henniger MS, Ohl F, Kuhne C, Putz B et al (2008). Profiling of behavioral changes and hippocampal gene 
expression in mice chronically treated with the SSRI paroxetine. Psychopharmacology (Berl) 200: 557-572.

Sukoff Rizzo SJ, Neal SJ, Hughes ZA, Beyna M, Rosenzweig-Lipson S, Moss SJ et al (2012). Evidence for sustained elevation of IL-6 in the CNS as a key contributor of depressive-like phenotypes. Transl Psychiatry 2: e199.

Surget A, Tanti A, Leonardo ED, Laugeray A, Rainer Q, Touma C et al (2011). Antidepressants recruit new neurons to improve stress response regulation. Mol Psychiatry 16: $1177-1188$.

Surget A, Wang Y, Leman S, Ibarguen-Vargas Y, Edgar N, Griebel G et al (2009). Corticolimbic transcriptome changes are statedependent and region-specific in a rodent model of depression and of antidepressant reversal. Neuropsychopharmacology 34: $1363-1380$.

Sweatt JD (2004). Mitogen-activated protein kinases in synaptic plasticity and memory. Curr Opin Neurobiol 14: 311-317.
Takahashi Y, Washiyama K, Kobayashi T, Hayashi S (2006). Gene expression in the brain from fluoxetine-injected mouse using DNA microarray. Ann NY Acad Sci 1074: 42-51.

Tanti A, Belzung C (2013). Neurogenesis along the septo-temporal axis of the hippocampus: are depression and the action of antidepressants region-specific? Neuroscience 252: 234-252.

Tardito D, Molteni R, Popoli M, Racagni G (2012). Synergistic mechanisms involved in the antidepressant effects of agomelatine. Eur Neuropsychopharmacol 22(Suppl 3): S482-S486.

Villanueva R (2013). Neurobiology of major depressive disorder. Neural Plast (Article ID 873278).

Wainwright SR, Galea LA (2013). The neural plasticity theory of depression: assessing the roles of adult neurogenesis and PSANCAM within the hippocampus. Neural Plast (Article ID 805497).

Willner P, Scheel-Kruger J, Belzung C (2013). The neurobiology of depression and antidepressant action. Neurosci Biobehav Rev 37(Pt 1): 2331-2371.

Supplementary Information accompanies the paper on the Neuropsychopharmacology website (http://www.nature.com/npp) 